\title{
Comportement de différentes accessions améliorées de féverole (Vicia faba L.) vis-à-vis d'Orobanche crenata Forsk. et Orobanche foetida Poir. ${ }^{*}$
}

\section{Zouhaier Abbes ${ }^{1}$ \\ Mohamed Kharrat ${ }^{1}$ \\ Khalil Shaaban ${ }^{2}$ \\ Bassam Bayaa ${ }^{2}$}

${ }^{1}$ Institut national de la recherche agronomique de Tunisie

Rue Hédi Karray

2080 Ariana

Tunisie

<zouhaier.abbes@fst.rnu.tn>

$<$ kharrat.mohamed@iresa.agrinet.tn>

$<$ k.shaaban@cgiar.org >

$<$ b.bayaa@cgiar.org>

2 ICARDA

PO Box 5466

Alep

Syrie

\begin{abstract}
Résumé
Huit accessions améliorées de féverole de la Pépinière internationale de féveroles pour la résistance à l'orobanche (FBION) du Centre international de la recherche agricole dans les régions arides (ICARDA) ont été étudiées dans des conditions naturelles d'infestation par les deux plantes parasites Orobanche foetida et Orobanche crenata dans les régions respectives de Béja et de l'Ariana (Tunisie), pendant les trois campagnes agricoles de 2002-2003, 2003-2004 et 2004-2005. L'objectif était d'évaluer leur comportement vis-à-vis des deux espèces de parasites en déterminant l'incidence et la sévérité de l'infestation, ainsi que la productivité moyenne en grains des féveroles. Tous les caractères étudiés ont montré de larges variations. Quelques accessions améliorées se sont mieux comportées que la lignée sensible incluse dans l'essai en tant que témoin. Ces accessions ont présenté un nombre faible d'orobanches émergées et un rendement plus élevé que celui du témoin sensible, montrant ainsi une bonne adaptation aux conditions climatiques locales. Elles peuvent ainsi être utilisées comme une composante essentielle dans la lutte intégrée contre l'orobanche et comme source de résistance afin de créer de nouvelles variétés résistantes.
\end{abstract}

Mots clés : Orobanche crenata ; Orobanche foetida ; résistance ; sélection ; Tunisie ; Vicia faba.

Thèmes : pathologie ; productions végétales.

\section{Abstract \\ Behaviour of different faba bean (Vicia faba L.) improved accessions in relation to Orobanche crenata Forsk. and Orobanche foetida Poir.}

Eight faba bean accessions from the Faba Bean International Orobanche Nursery distributed by the International Center of Agricultural Research in the Dry Areas (ICARDA) were studied in fields naturally infested by Orobanche feotida and Orobanche crenata, in the Beja (Orobanche feotida) and Ariana (Orobanche crenata) regions (Tunisia) during the 2002-2003, 2003-2004 and 2004-2005 seasons. The objective was to evaluate their behavior in the presence of the two Orobanche species by determining the incidence and the severity of infestation and grain yield. All the characters studied showed broad variations. Some improved accessions behaved better than the susceptible line included in the essays as control. These improved accessions presented lower numbers of emerged broomrape and a higher seed yield compared to the controls. These accessions can form appropriate material for an integrated control package and can be used as sources of resistance to create new varieties resisting Orobanche.

Key words: Orobanche crenata; Orobanche foetida; resistance; selection; Tunisia; Vicia faba.

Subjects: pathology; vegetal productions. 
L' espèce Vicia faba L., l'une des plus anciennes légumineuses alimentaires domestiquées dans le monde, joue un rôle important dans les systèmes agricoles des pays méditerranéens. Elle n'est pas seulement importante pour la nutrition humaine, mais contribue également à la nutrition animale, tout en ayant un effet positif sur la productivité des céréales qui font partie de la rotation (Saxena, 1991).

Les attaques par la plante parasite Orobanche spp. comptent parmi les principales maladies affectant cette culture. La présence de cet agent pathogène occasionne des pertes considérables pouvant entraîner la destruction totale de la culture (Abbes et al., 2007). Les pertes de rendement varient néanmoins d'un pays à l'autre et d'une année à l'autre dans la même parcelle. Mesa Garcia et Garcia Torres (1986) ont rapporté des pertes allant de 5 à $95 \%$ sur la fève.

La plus grande diversité des espèces d'orobanches se trouve dans la région méditerranéenne. La plante parasite la plus redoutable sur les légumineuses et quelques autres cultures dans la région méditerranéenne est Orobanche crenata, présente principalement en Afrique du Nord, dans le Sud-Est de l'Espagne, le Sud de l'Italie, et dans quelques régions de Grèce, de Syrie et d'Égypte.

L'espèce Orobanche foetida est endémique à ce jour de l'Afrique du Nord et du Sud de l'Espagne, où elle est signalée essentiellement comme parasite de légumineuses non cultivées. L'écotype tunisien semble être le plus virulent, car il s'attaque à certaines légumineuses cultivées, comme les fèves (Abbes et al., 2007). Récemment, Rubiales et al. (2005) ont signalé l'infestation de parcelles de Vicia sativa par O. foetida au Maroc, ce qui souligne le risque d'une dispersion d'écotypes virulents de cette espèce d'orobanche dans les régions aux conditions climatiques comparables.

Plusieurs méthodes de lutte (culturales, chimiques, entre autres) contre l'orobanche ont été testées, mais aucune n'est, à elle seule, complètement efficace, économique et facile à mettre en œuvre. La stratégie la plus prometteuse est l'amélioration et l'utilisation de variétés tolérantes de $V$. faba en combinaison avec les autres méthodes. En Égypte, Nassib et al. (1982) ont sélectionné une lignée de féverole résistante à $O$. crenata. Cette lignée (F402) fut ainsi la première lignée cumulant un bon niveau de résistance et des caractères agronomiques intéressants. Sa multiplication a abouti à la production du cultivar Giza 402, dont la résistance a été caractérisée sous diverses conditions. Cette lignée fut utilisée avec succès à grande échelle comme source de résistance à l'orobanche dans différents programmes de sélection de la fève (Maroc, Égypte, Espagne, Tunisie, Centre international de la recherche agricole dans les régions arides [International Center of Agricultural Research in the Dry Areas] en Syrie) (Khalil et al., 2004 ; Abbes et al., 2007).

L'objectif de cette étude est de vérifier dans des parcelles naturellement infestées par O. foetida et $O$. crenata la résistance de quelques accessions améliorées de féverole introduites de l'International Center of Agricultural Research in the Dry Areas (ICARDA) et sélectionnées en Égypte et en Syrie pour leur tolérance à O. crenata.

\section{Matériel et méthode}

\section{Matériel végétal}

Les différentes accessions améliorées de féverole utilisées ont été fournies par l'ICARDA à Alep (Syrie), dans la pépinière internationale pour l'évaluation des fèves vis-à-vis de l'orobanche FBION (Faba Bean International Orobanche Nursery) (tableau 1). Le matériel végétal testé englobe les accessions améliorées de féveroles les plus prometteuses sélectionnées pour leur résistance à $O$. crenata en Égypte et en Syrie à l'ICARDA (Khalil et al., 2004). Un témoin sensible (ILB1814) est utilisé. Les accessions améliorées proviennent de sélection de plantes individuelles à partir de croisements réalisés en Egypte incluant des parents tolérants à l'orobanche, à d'autres maladies fongiques et possédant des caractères agronomiques d'intérêt (précocité, productivité, etc.) (Khalil et al., 2004).

\section{Dispositif expérimental}

Les accessions de féveroles ont été cultivées dans deux parcelles naturellement infestées par O. foetida à la station d'Oued Béja (latitude $36^{\circ} 43^{\prime} \mathrm{N}$, longitude $9^{\circ} 12^{\prime} \mathrm{E}$ ) et par $O$. crenata à la station de l'Ariana (latitude $36^{\circ} 50^{\prime} \mathrm{N}$, longitude $10^{\circ} 11^{\prime} \mathrm{E}$ ) de l'Institut national de la recherche agronomique de Tunisie (Inrat). Les expérimentations ont été conduites sur trois années, durant les campagnes 2002-2003, 20032004 et 2004-2005. Les conditions climatiques (température et pluviométrie) pour les trois saisons sont présentées dans la figure 1.

\section{Tableau 1. Matériel végétal utilisé dans cette étude. Les accessions sont issues de la pépinière internationale de féverole pour la résistance à l'orobanche (FBION) de I'ICARDA (International Center of Agricultural Research in the Dry Areas).}

Table 1. Genetic material used in this study. Faba bean accessions from the Faba Bean International Orobanche Nursery (FBION) of ICARDA.

\begin{tabular}{|c|c|c|c|}
\hline Accession & Croisement/pedigree & $\begin{array}{l}\text { Principale } \\
\text { caractéristique }\end{array}$ & Origine \\
\hline 1013/694/95 & X-1013 : (Giza 3 x ILB938) × 30/18/82 & Précoce & Égypte \\
\hline 1016/752/95 & $\begin{array}{l}X-1016:(461 / 842 / 83 \times 503 / 453 / 83) \times \\
30 / 18 / 82\end{array}$ & Précoce & Égypte \\
\hline $985 / 252 / 95$ & $\begin{array}{l}\text { X-985: }(461 / 842 / 83 \times 503 / 453 / 83) \times \\
\text { (Giza } 402 \times \text { ILB938) }\end{array}$ & Précoce & Égypte \\
\hline 989/303/95 & $\begin{array}{l}\text { X-989 : Giza } 402 \text { x (Giza } 402 \text { x } \\
\text { ILB938) }\end{array}$ & Précoce & Égypte \\
\hline 989/306/95 & $\begin{array}{l}\text { X-989: Giza } 402 \text { x (Giza } 402 \text { x } \\
\text { ILB938) }\end{array}$ & Précoce & Égypte \\
\hline 989/309/95 & $\begin{array}{l}\text { X-989: Giza } 402 \text { x (Giza } 402 \text { x } \\
\text { ILB938) }\end{array}$ & Précoce & Égypte \\
\hline cv. Giza 4 & ILB1820: Giza 1 x NA29 & Précoce & Égypte \\
\hline Témoin sensible & ILB1814 : population locale syrienne & Précoce & Syrie \\
\hline
\end{tabular}



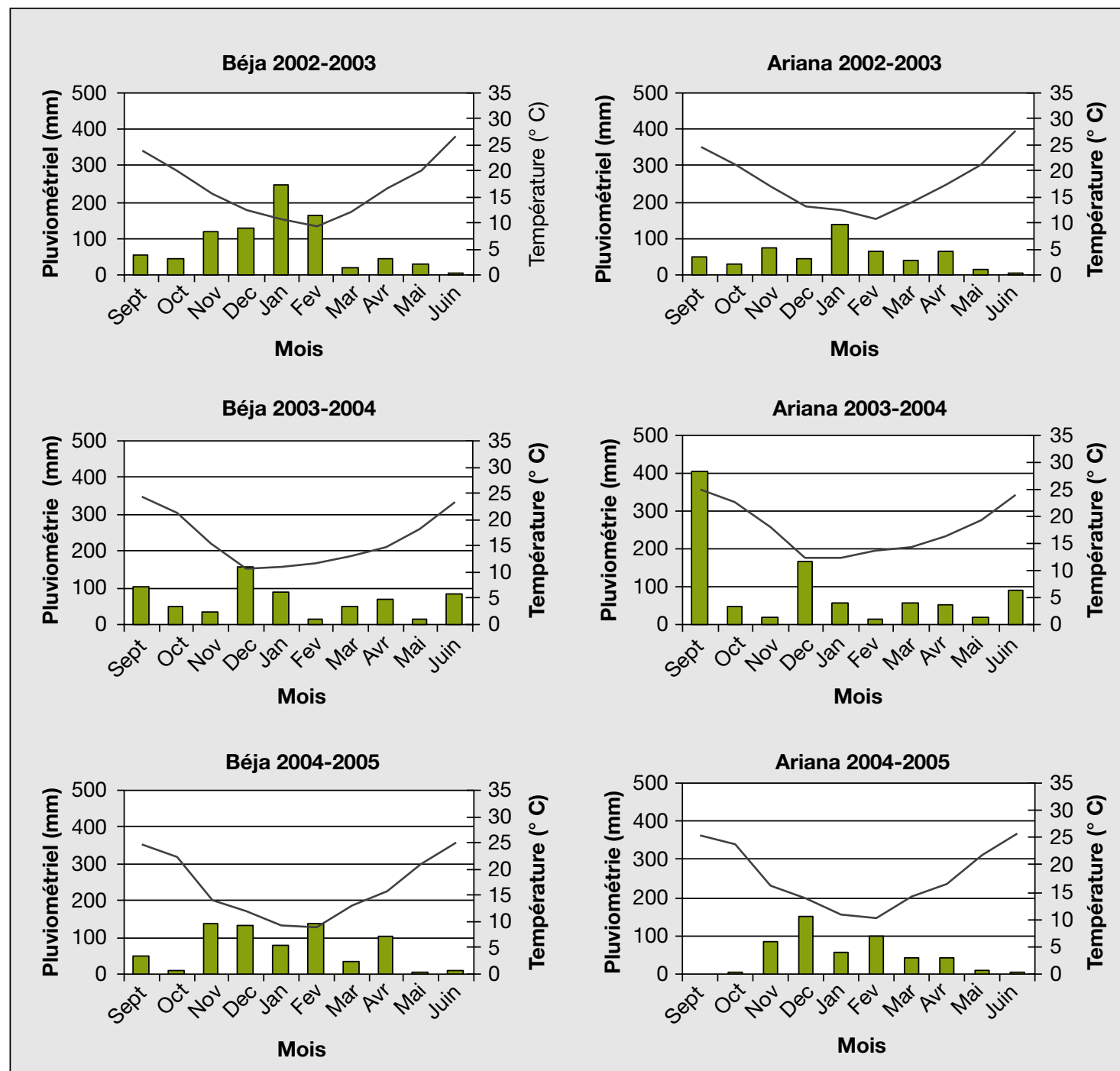

Figure 1. Répartition de la pluviométrie (histogramme) et de la température (-, courbe) durant les trois campagnes agricoles à Oued Béja et à l'Ariana.

Figure 1. Distribution of rainfall (histogram) and temperature (-, curve) during the three cropping seasons at Oued Beja and Ariana.

Le dispositif expérimental utilisé consiste en blocs complets randomisés avec trois répétitions dans les deux sites, à l'exception des essais de l'Ariana en 2002-2003 et 2004-2005 où deux répétitions seulement sont semées. Pour les deux essais, chaque accession est semée, à raison de 20 graines, sur une ligne de 4 et 3,5 mètres de longueur et un écartement de 0,5 et 0,4 mètre entre les lignes, pour respectivement la station d'Oued Béja et celle de l'Ariana. Le témoin sensible (ILB1814) est inséré toutes les deux lignes dans les deux essais.

Les semis sont réalisés durant la période qui va de la fin novembre au début décembre. Deux traitements herbicides sont appliqués pour le contrôle des adventices : trifluraline (960 g de matière active par hectare (m.a./ha) avant le semis, et simazine (720 g m.a./ha) avant la levée de la culture. Ces traitements n'ont pas d'effets sur les attaques d'orobanche.

\section{Paramètres mesurés}

L'évaluation de la résistance et de la sensibilité des accessions a porté sur les paramètres suivants, mesurés à maturité (récolte des graines de féverole):

- le nombre d'orobanches émergées par plante (NOEP) ;
- la sévérité, estimée visuellement suivant une échelle de 1 à 9, 1 représentant un développement normal de la plante hôte avec absence ou quasi-absence de pieds d'orobanches émergées (haute résistance), et 9 représentant une réduction très prononcée du rendement ou destruction de la plante hôte avec une présence importante du parasite (haute sensibilité) (Abbes et al., 2007) ;

- l'incidence qui représente le pourcentage des plantes présentant des orobanches émergées (0\%: absence d'orobanche émergée, et $100 \%$ : toutes les plantes de féverole présentent des orobanches émergées) ;

- le rendement en $\mathrm{g} /$ plante. 
Méthodes d'analyses statistiques

L'analyse de la variance est effectuée en adoptant deux critères de classification (effets accession et bloc) pour les données de chaque site et de chaque campagne. Une analyse des données des trois années de chaque site est également réalisée pour déterminer l'effet année, l'effet accession et l'interaction accession-année. La comparaison des moyennes est faite en utilisant le test de Duncan $(P=0,05)$. Les analyses statistiques sont réalisées avec le logiciel $S A S^{\circledR}$ (Statistical Analysis system), version 8.01 (Institut SAS, 2000).

\section{Résultats}

L'étude a montré que le nombre d'orobanches émergées par plante (NOEP) est nettement plus élevé dans la station de l'Ariana que dans celle d'Oued Béja au cours des campagnes 2002-2003 et 20032004. En revanche, durant la campagne 2004-2005, les deux niveaux d'infestation sont comparables (tableau 2).

Dans les deux sites expérimentaux et durant les trois campagnes, les conditions climatiques ont été en général favorables pour le développement de la culture et pour une bonne infestation par l'orobanche, cela en raison d'une pluviométrie plus ou moins élevée et d'une température modérée (figure 1). Le faible niveau d'infestation observé à l'Ariana durant l'année 2004-2005 pourrait être dû au semis relativement tardif de l'essai (début décembre).

$\mathrm{Au}$ cours d'une même année, une grande variabilité est constatée entre les accessions pour le paramètre NOEP (tableau 2). Cependant, cette variabilité ne conduit pas toujours à l'expression de différences significatives entre les accessions (cas de la campagne 20022003 pour O. crenata), bien qu'un nombre plus élevé d'orobanches émergées soit enregistré à maturité au niveau du témoin sensible (ILB1814). L'analyse des données du caractère NOEP des trois années ne montre un effet année significatif $(P=0,029)$ que dans le site de l'Ariana, tandis que l'effet accession était hautement significatif pour les deux sites. Les interactions accession-année dans les deux sites n'étaient pas significatives. La moyenne des résultats des trois années montre une différence significative pour ce paramètre entre le témoin sensible ILB1814 et les autres accessions pour $O$. foetida et pour O. crenata.

Dans cette étude, l'incidence a atteint des valeurs élevées, très proches de $90 \%$, pour le témoin sensible ILB1814 et des valeurs plus faibles pour les autres accessions améliorées, mais aucune différence significative n'est déterminée pour ces accessions dans l'analyse des données par site et par année (tableau 3). Cependant, l'analyse des données des trois campagnes des essais réalisés à l'Ariana a révélé que les effets année, accession et leur interaction sont significatifs $(0,05>\mathrm{P}>0,01)$. Ce résultat est conséquence de la faible incidence observée durant la campagne 2004-2005 sur ce site. L'estimation du paramètre sévérité (tableau 4) a permis la distinction entre le témoin sensible (ILB1814) et les autres accessions, notamment dans les essais réalisés à Oued Béja. L'analyse des données des trois campagnes a montré un effet année significatif dans les deux sites. L'effet accession n'est, en revanche, significatif que pour le site d'Oued Béja. Les interactions accession-année, quant à elles, n'étaient pas significatives dans les deux sites.

L'analyse des données des trois campagnes du paramètre rendement par plante a montré que l'effet année est significatif $(\mathrm{P}=0,049)$ dans le site de Béja et hautement significatif $(P=0,008)$ dans le site de l'Ariana, tandis que l'effet accession ne l'est pas dans les deux sites. L'interaction accession-année n'est significative $(\mathrm{P}=0,015)$ que dans la parcelle de l'Ariana. Dans cette parcelle, les accessions les plus productives sont 985/252/95, 989/306/95 et 1013/694/95, et ce bien qu'elles portent un nombre élevé d'orobanches émergées (tableau 5). En revanche, dans la parcelle infestée d'Oued Béja, les accessions 989/303/95, $989 / 309 / 95$ et $1016 / 752 / 95$ sont les plus productives.

\section{Discussion}

Les principaux objectifs de ces essais au champ étaient d'évaluer la résistance de différentes accessions de féveroles à $O$. foetida et $O$. crenata. Dans le cadre des programmes de sélection, l'appréciation au champ du degré de résistance aux plantes parasites (Orobanche et Striga) a nécessité la définition d'indicateurs de

\section{Tableau 2. Nombre de pieds d'orobanche émergées/plante (NOEP) pour les différentes accessions de féverole à Oued Béja et à l'Ariana}

Table 2. Number of emerged broomrape shoots/plant (NOEP) of the different faba bean accessions evaluated at Beja and Ariana.

\begin{tabular}{|c|c|c|c|c|c|c|c|c|}
\hline \multirow[t]{2}{*}{ Accession } & \multicolumn{3}{|c|}{ O. foetida-Oued Béja } & \multirow[t]{2}{*}{ Moyenne } & \multicolumn{3}{|c|}{ O. crenata-Ariana } & \multirow[t]{2}{*}{ Moyenne } \\
\hline & 2002-2003 & 2003-2004 & 2004-2005 & & 2002-2003 & 2003-2004 & 2004-2005 & \\
\hline 1013/694/95 & $2,3 a b^{*}$ & $2,5 a b c$ & $1,9 a$ & $2,3 a b$ & $11,8 a$ & $14,0 a b c$ & $1,4 a$ & $9,8 a b$ \\
\hline 1016/752/95 & $1,9 a$ & $1,0 a$ & $2,0 a$ & $1,6 a$ & $16,0 a$ & $16,6 c$ & $0,9 a$ & $11,9 b c$ \\
\hline 985/252/95 & $1,9 a$ & $2,1 a b$ & $2,4 a$ & $2,1 \mathrm{ab}$ & $13,3 a$ & $12,5 a b c$ & $0,9 a$ & $9,4 a b$ \\
\hline 989/303/95 & $3,6 b$ & $2,0 a b$ & $2,1 \mathrm{a}$ & $2,6 b$ & $12,0 a$ & $12,8 a b c$ & $1,0 a$ & $9,2 \mathrm{a}$ \\
\hline 989/306/95 & $3,2 a b$ & $1,9 a b$ & $2,9 a b$ & $2,7 \mathrm{~b}$ & $12,5 a$ & $11,1 \mathrm{a}$ & $0,9 a$ & $8,6 a$ \\
\hline 989/309/95 & $2,5 a b$ & $2,1 \mathrm{ab}$ & $1,4 a$ & $2,0 a b$ & $14,9 a$ & $10,2 a$ & $1,4 a$ & $9,0 a$ \\
\hline Giza 4 & $2,1 \mathrm{a}$ & $2,2 a b$ & $1,9 a$ & $2,0 \mathrm{ab}$ & $8,5 a$ & $12,3 a b$ & $0,9 a$ & 8,0 a \\
\hline Témoin sensible ILB1814 & $6,3 c$ & $4,7 \mathrm{bc}$ & $4,3 b$ & $5,1 \mathrm{c}$ & $17,1 \mathrm{a}$ & $15,9 b c$ & $3,4 b$ & $12,7 \mathrm{c}$ \\
\hline
\end{tabular}

* Pour chaque colonne, les valeurs suivies de la même lettre ne diffèrent pas significativement. 
Tableau 3. Incidence de l'infestation (\%) en présence des différentes accessions de féverole testées à Oued Béja et à l'Ariana

Table 3. Incidence of infestation (\%) in the presence of the different faba bean accessions evaluated at Beja and Ariana.

\begin{tabular}{|c|c|c|c|c|c|c|c|c|}
\hline \multirow[t]{2}{*}{ Accession } & \multicolumn{3}{|c|}{ O. foetida-Oued Béja } & \multirow[t]{2}{*}{ Moyenne } & \multicolumn{3}{|c|}{ O. crenata-Ariana } & \multirow[t]{2}{*}{ Moyenne } \\
\hline & 2002-2003 & 2003-2004 & 2004-2005 & & 2002-2003 & 2003-2004 & 2004-2005 & \\
\hline 1013/694/95 & $67 a^{*}$ & $82 a$ & $58 a$ & $69 a$ & $100 a$ & $100 \mathrm{a}$ & $35 a$ & $81 a$ \\
\hline 1016/752/95 & $48 a$ & $73 a$ & $47 a$ & $56 a$ & $100 a$ & $100 a$ & $25 a$ & $79 a$ \\
\hline 985/252/95 & $53 a$ & $73 a$ & $87 a$ & $71 a$ & $100 a$ & $100 \mathrm{a}$ & $40 a$ & $83 a b$ \\
\hline 989/303/95 & $82 a$ & $87 a$ & $50 a$ & $73 a$ & $100 a$ & $100 a$ & $25 a$ & $79 a$ \\
\hline 989/306/95 & $68 a$ & $73 a$ & $67 a$ & $69 a$ & $100 a$ & $100 \mathrm{a}$ & $35 a$ & $81 a$ \\
\hline 989/309/95 & $62 a$ & $78 a$ & $40 a$ & $60 a$ & $100 a$ & $100 \mathrm{a}$ & $50 a$ & $86 a b$ \\
\hline Giza 4 & $68 a$ & $82 a$ & $60 a$ & $70 a$ & $100 \mathrm{a}$ & $100 \mathrm{a}$ & $40 a$ & $83 a b$ \\
\hline Témoin sensible ILB1814 & $94 a$ & $93 a$ & $77 a$ & $88 a$ & $99 a$ & $100 a$ & $69 a$ & 91b \\
\hline
\end{tabular}

* Pour chaque colonne, les valeurs suivies de la même lettre ne diffèrent pas significativement.

\section{Tableau 4. Sévérité de l'infestation en présence des différentes accessions de féverole testées} à Oued Béja et à I'Ariana

Table 4. Severity of infestation in presence of the different faba bean accessions evaluated at Beja and Ariana.

\begin{tabular}{|c|c|c|c|c|c|c|c|c|}
\hline \multirow[t]{2}{*}{ Accession } & \multicolumn{3}{|c|}{ O. foetida-Oued Béja } & \multirow[t]{2}{*}{ Moyenne } & \multicolumn{3}{|c|}{ O. crenata-Ariana } & \multirow[t]{2}{*}{ Moyenne } \\
\hline & 2002-2003 & 2003-2004 & $2004-2005$ & & $2002-2003$ & 2003-2004 & 2004-2005 & \\
\hline 1013/694/95 & $4,3 a b^{*}$ & $6,3 a b$ & $3,7 a$ & $4,8 a$ & $9,0 a$ & $7,3 a b$ & $3,0 a$ & $6,6 a$ \\
\hline 1016/752/95 & $3,3 a$ & $6,0 a$ & $4,0 \mathrm{a}$ & $4,4 a$ & $9,0 a$ & $6,7 a b$ & $2,5 a$ & $6,1 a$ \\
\hline $985 / 252 / 95$ & $3,7 a$ & $5,7 a$ & $4,3 a$ & $4,6 a$ & $9,0 a$ & $5,3 a$ & $3,0 a$ & $5,7 a$ \\
\hline 989/303/95 & $5,3 b c$ & $6,7 a b c$ & $4,0 a$ & $5,3 a$ & $9,0 a$ & $6,3 a b$ & $2,0 a$ & $5,9 a$ \\
\hline 989/306/95 & $6,3 \mathrm{~cd}$ & $7,7 c$ & $4,7 a b$ & $6,2 b$ & $9,0 a$ & $7,0 a b$ & $2,5 a$ & $6,3 a$ \\
\hline 989/309/95 & $5,0 a b c$ & $7,3 \mathrm{bc}$ & $3,3 a$ & $5,2 a$ & $9,0 a$ & $6,3 a b$ & $3,5 a$ & $6,3 a$ \\
\hline Giza 4 & $4,3 a b$ & $6,3 a b$ & $4,7 a b$ & $5,1 a$ & $8,0 a$ & $6,7 a b$ & $3,0 a$ & $6,0 \mathrm{a}$ \\
\hline Témoin sensible ILB1814 & $7,7 d$ & $7,7 c$ & $6,4 b$ & $7,3 \mathrm{c}$ & $8,9 a$ & $8,3 b$ & $4,2 a$ & $7,3 \mathbf{a}$ \\
\hline
\end{tabular}

* Pour chaque colonne, les valeurs suivies de la même lettre ne diffèrent pas significativement.

Tableau 5. Rendement (g/plante) des différentes accessions de féverole testées à Oued Béja et à l'Ariana Table 5. Grain yield (g/plant) of the different faba bean accessions evaluated at Beja and Ariana.

\begin{tabular}{|c|c|c|c|c|c|c|c|c|}
\hline \multirow[t]{2}{*}{ Accession } & \multicolumn{3}{|c|}{ O. foetida-Oued Béja } & \multirow[t]{2}{*}{ Moyenne } & \multicolumn{3}{|c|}{ O. crenata-Ariana } & \multirow[t]{2}{*}{ Moyenne } \\
\hline & $2002 / 03$ & $2003 / 04$ & 2004/05 & & $2002 / 03$ & $2003 / 04$ & $2004 / 05$ & \\
\hline 1013/694/95 & $8,2 a b^{*}$ & $4,3 a b$ & $7,6 a b$ & $6,7 a$ & $0,8 a$ & $3,8 a b$ & $17,3 a$ & $6,8 a$ \\
\hline 1016/752/95 & $8,3 a b$ & $2,7 a$ & $9,9 b$ & $7,0 a$ & $0,2 a$ & $3,8 a b$ & $16,8 a$ & $6,5 a$ \\
\hline 985/252/95 & $7,6 a b$ & $5,5 a b$ & $6,6 a b$ & $6,6 a$ & $0,0 a$ & $6,8 b$ & $21,0 a$ & $8,9 a$ \\
\hline 989/303/95 & $7,0 a b$ & $4,5 a b$ & $10,8 b$ & $7,4 a$ & $0,1 \mathrm{a}$ & $3,5 a b$ & $16,2 a$ & $6,2 a$ \\
\hline 989/306/95 & $4,7 a b$ & $3,4 a b$ & 7,9ab & $5,4 a$ & $0,1 a$ & $5,8 b$ & $19,5 a$ & $8,1 a$ \\
\hline 989/309/95 & $11,8 b$ & $3,1 \mathrm{ab}$ & $7,1 \mathrm{ab}$ & $7,4 a$ & $0,0 a$ & $3,9 a b$ & $12,2 a$ & $5,2 a$ \\
\hline Giza 4 & $6,2 a b$ & $6,1 b$ & $5,3 a$ & $5,9 a$ & $1,6 a$ & $4,3 a b$ & $8,8 a$ & $4,8 a$ \\
\hline Témoin sensible ILB1814 & $2,0 a$ & $2,4 a$ & $7,8 a b$ & $4,1 \mathrm{a}$ & $0,4 a$ & $0,3 a$ & $21,5 a$ & $6,4 a$ \\
\hline
\end{tabular}

* Pour chaque colonne, les valeurs suivies de la même lettre ne diffèrent pas significativement. 
résistance simples et faciles à quantifier. Différents paramètres quantitatifs ont été ainsi utilisés selon les auteurs : le nombre d'orobanches émergées par plante, la masse sèche d'orobanches émergées par plante, la longueur moyenne de la tige du parasite, etc. (Rubiales et al., 2006). Le nombre d'orobanches émergées par plante est l'indice le plus couramment utilisé (Rubiales et al., 2003). Dans cette étude, ce paramètre a permis de distinguer entre accessions tolérantes et sensibles. Néanmoins, d'après Sillero et al. (1996), fonder une sélection exclusivement sur le nombre d'orobanches émergées par plant est risqué. La vigueur et la productivité de la plante hôte doivent être également prises en considération. Ainsi, dans notre étude, des indices agronomiques incluant le nombre d'orobanche par plante hôte, la vigueur et la productivité de la plante infectée ont été définis, tels que l'incidence et la sévérité. Le paramètre sévérité a permis la distinction entre les accessions tolérantes et sensibles. Les valeurs élevées de l'incidence montrent que la distribution des graines d'orobanche semblerait être uniforme dans les parcelles utilisées, ce qui permet d'avoir une assez bonne évaluation du comportement des accessions évaluées.

Dans notre étude, les différentes accessions utilisées ont présenté une moindre sensibilité aux deux plantes parasites $O$. crenata et $O$. foetida, avec une augmentation du rendement jusqu'à $82 \%$ pour O. foetida et $39 \%$ pour O. crenata par rapport au témoin sensible. Ces mêmes accessions, testées en Syrie (ICARDA) vis-à-vis d'O. crenata, ont montré un nombre de pieds d'orobanche faible, mais leur rendement a été négativement affecté par le parasitisme par rapport au témoin sensible (Khalil et al., 2004). Il est évident que la résistance présentée est une résistance partielle, affectée par l'environnement, comme suggéré par plusieurs auteurs (Rubiales et al., 2003; Radwan et al., 1988). Les travaux de sélection doivent, par conséquent, être concentrés sur la recherche de génotypes combinant une bonne vigueur, un bon rendement avec un nombre d'orobanches faible et une faible interaction avec l'environnement.

Dans les deux sites expérimentaux, les accessions les plus productives montrent à leurs pieds un nombre relativement élevé d'orobanches émergées. Cette constatation suggère que ces accessions sont tolérantes plutôt que résistantes. Cubero et Hernandez (1991) ont démontré le caractère multigénique avec des effets additifs importants de la résistance de la lignée F402, à l'origine de la variété Giza402. Cette résistance partielle est plutôt de type horizontale. La coexistence de plusieurs mécanismes de résistance induit une hétérogénéité dans le comportement parfois différent en fonction de l'environnement et de l'espèce d'orobanche.

Dans cette étude, la tolérance de certaines accessions à $O$. foetida atteste de l'efficacité des sources de tolérance à $O$. crenata vis-à-vis d'O. foetida et ainsi du transfert et de l'utilisation possibles en sélection de ces dernières pour la création de génotypes de féveroles résistants à $O$. foetida. Ainsi, la lignée tunisienne XBJ90.03-16-1-1-1, récemment sélectionnée à l'Inrat pour sa résistance à O. foetida, est le résultat d'un croisement entre une lignée locale et une lignée tolérante à $O$. crenata, produite par ICARDA et dont la source de résistance provient de la lignée égyptienne Giza 402, résistante à O. crenata (Abbes et al., 2007).

La tolérance observée chez différentes accessions de féverole à ces deux espèces d'orobanches laisse penser que celle-ci pourrait s'exprimer vis-à-vis d'autres écotypes d'orobanche aussi bien $O$. crenata qu'O. foetida. Cependant, étant donné le système de reproduction des fèves tendant vers l'allogamie et le rôle important des insectes pollinisateurs dans la fécondation (Holden et Bond, 1960), il est nécessaire de garder un isolement adéquat lors de la multiplication de ces accessions, afin de conserver leurs gènes de résistance.

\section{Conclusion}

Les sept accessions améliorées testées présentent un certain niveau de tolérance aux deux plantes parasites $O$. foetida et O. crenata. Leur résistance n'est pas pour autant totale et ces accessions améliorées, utilisées seules comme moyen de lutte, ne résolvent pas le problème de la contamination exponentielle des sols en graines du parasite. Elles peuvent néanmoins être utilisées comme une composante essentielle dans la lutte intégrée contre l'orobanche et comme source de résistance pour la création d'autres génotypes résistants et productifs.

\section{Références}

Abbes Z, Kharrat M, Delavault P, Simier P, Chaibi $W$. Field evaluation of the resistance of some faba bean (Vicia faba L.) lines to the parasitic weed Orobanche foetida Poiret. Crop Prot 2007 ; 26 : 1777-84.

Cubero JI, Hernandez L. Breeding faba bean (Vicia faba) for resistance to Orobanche crenata Forsk. Options Méditerranéennes, Ser Séminaires $1991 ; 10: 51-7$.

Holden JHW, Bond DA. Studies on the breeding system of the field bean, Vicia faba (L.). Heredity $1960 ; 15: 175-92$.

Institut SAS. Statistical Analysis System (SAS) pour Windows. Version 8.01. Cary (Caroline du nord) : Institut SAS, 2000.

Khalil S, Kharrat M, Malhotra R, Saxena M, Erskine W. Breeding faba bean for Orobanche resistance. In: Dahan R, El-Mourid M, eds. Integrated Management of Orobanche in Food Legumes in the Near East and North Africa. Proceedings of the Expert Consultation on IPM for Orobanche in Food Legume Systems in the Near East and North Africa. Rabbat, Morocco, 2004. Alep : ICARDA, 2004.

Mesa-Garcia J, Garcia-Torres L. Effect of planting date on parasitism of broad bean (Vicia faba) by crenate broomrape (Orobanche crenata). Weed Sci 1986 ; $34: 544-50$.

Nassib AM, Ibrahim AA, Khalil SA. Breeding for resistance to Orobanche. In: Hawtin G, Webb C, eds. Faba Bean Improvements. La Haye : Martinus Nijhoff, 1982.

Radwan MS, Abdalla MMF, Fischbeck G, Metwally AA, Darwish DS. Selection in faba bean for tolerance to broomrape, Orobanche crenata Forsk. Plant Breed 1988 ; 101 : 208-16.

Rubiales D, Perez-de-Luque A, Cubero JI, Sillero JC. Crenate broomrape (Orobanche crenata) infection in field pea cultivars. Crop Prot 2003 $22: 865-72$.

Rubiales D, Sadiki M, Romero B. First report of Orobanche foetida on common vetch (Vicia sativa) in Morocco. Plant Dis 2005 ; 89 : 528.

Rubiales D, Perez-de-Luque A, FernandezAparicio $M$, et al. Screening techniques and sources of resistance against parasitic weeds in grain legumes. Euphytica 2006 ; 147 : 187-99.

Saxena MC. Status and scope for production of faba bean in the Mediterranean countries. Options Méditerranéennes, Ser. Séminaires $1991 ; 10: 15-20$

Sillero JC, Rubiales D, Cubero JI. Risk of orobanche resistance screening based only on number of emerged shoots per plant. In: Moreno MT, Cubero Jl, Berner D, Joel D, Musselman LJ, Parker $\mathrm{C}$, eds. Advances in parasitic plant research. Proceedings of the 6th International on parasitic weed symposium, Cordoba, Espagne, 1996. Séville : AG Novograf, 1996 